\title{
The Effects of Proprioceptive Neuromuscular Facilitation and Stabilizing Exercise on Trunk Repositioning Errors
}

\author{
Sookhee Byuon, PhD, $\mathrm{PT}^{1)}$, Hohee Son, PhD, $\mathrm{PT}^{2)}$ \\ 1) Department of Physical Therapy, Daegu Science College \\ 2) Department of Physical Therapy, College of Health Sciences Catholic University of Pusan: 57 \\ Oryundae-ro, Geumjeong-go, Busan, 609-757, South Korea. \\ TEL: +82 11-505-8065, FAX: +82 51-510-0578, E-mail: sonhh@cup.ac.kr
}

\begin{abstract}
Purpose] The purpose of this study was to evaluate trunk repositioning errors after PNF exercise, or stabilizing exercise for low back pain. [Methods] Subjects were randomly assigned to a PNF exercise group or a stabilizing exercise group. The PNF exercise group voluntarily participated in PNF programs for the low back stability 4 times a week for 6 weeks. The stabilizing exercise group took part in stabilizing exercises 4 times a week for 6 weeks. Outcome measures were subjective pain measured on a visual analogue scale (VAS), and trunk repositioning error (RE). [Results] The VAS score was significantly reduced by the intervention in the PNF and stabilizing groups, but the difference in VAS scores between the groups was not significant. The RE of the PNF and stabilizing groups was significantly reduced by the intervention and of the difference in the RE score between the groups was also significant. [Conclusion] Based on these results, we conclude that PNF exercise and stabilizing exercise both reduce subjective pain and RE. Therefore PNF exercise and stabilizing exercise are useful for improving the low back stability of low back pain patients. Accordingly we consider that PNF exercise will be beneficial for low back pain. Key words: PNF exercise, Repositioning error, Stabilizing exercise
\end{abstract}

(This article was submitted May 7, 2012, and was accepted Jun. 5, 2012)

\section{INTRODUCTION}

Human spines are composed of multiple joints, which have conflicting characteristics. Each vertebral joint has a small range of motion due to its limited movement, but interactions among the different segments enable a large total range of motion with structural stability and functional mobility. Eighty percent of humans experience lumbar pain at some point in their lives due to the fundamental instability of the spine. Lumbar pain is not only a medical problem but it has also emerged as a social and economic issue ${ }^{1)}$.

Lumbar pain reduces the activity of the cross-sections of muscles around the spine and weakens muscle strength, thereby bringing about disuse atrophy. Furthermore, it decreases endurance and flexibility, thereby limiting the range of lumbar motion ${ }^{2}$.

Diverse treatment methods are used to improve weakened muscle strength or damaged soft tissues resulting from lumbar pain. Previous studies have reported that exercise is effective as an aggressive treatment method. Cakir et al. pointed out the fundamental biomechanical problems of the lumbar spine, noting that the main etiology of the development and recurrence of lumbar pain was lumbar segmental instability ${ }^{3}$. A recent study reported that lumbar pain patients had weakened and unbalanced deep muscles and reduced proprioceptive capabilities, compared to normal people, resulting in increased joint repositioning errors and problems with spinal stability. These triggered continuous recurrence of lumbar pain ${ }^{4)}$.

Joint repositioning errors are the most common standard by which the proprioceptive senses are examined. Examining these errors is a test of the difference between the original target location and the location realized by a subject after establishing a target angle or location of the joints using the position or movement senses and then making the subject represent that location ${ }^{5}$.

The proprioceptive senses are used to adjust motor senses in all areas of the body, including the spine ${ }^{6)}$. These senses help us to perceive the degree of muscular contraction, muscle shapes, and the locations and movements of the joints. Proprioceptive senses have an afferent system, in which simulation is afferently delivered to the muscles, joints, and skin receptors ${ }^{7)}$. Multiple studies have reported that chronic lumbar pain patients have damaged position senses and postural adjustment functions ${ }^{8,9)}$. Accordingly, it is crucial to enhance lumbar pain patients' proprioceptive senses in order to cure lumbar pain and prevent its recurrence. Proprioceptive neuromuscular facilitation (PNF) techniques are known to be effective at improving proprioceptive function. They stimulate proprioceptors within the muscles and tendons, increasing muscle strength, flexibility, and balance and enhance coordination abilities in response to neuromuscular stimuli, enabling maximum motor unit response $\mathrm{e}^{10)}$. 
The PNF technique is a treatment method that is used to stimulate proprioceptors and facilitate normal responses. It promotes the response of the neuromuscular mechanism by stimulating the proprioceptive senses, such as the muscle spindles that produce afferent excitation ${ }^{11)}$.

Improving proprioceptive sense is most important for the stability of the lumbar spine. Accordingly, this study examined stabilization and PNF exercises that are used to enhance muscle strength and stabilize the lumbar region. Previous studies of the proprioceptive senses have focused on whether joints were able achieve target locations by conducting lumbar spine repositioning error tests on lumbar pain patients. However, in this study low back pain patients performed either stabilization exercise or PNF exercise, to provide data on repositioning error and to promote the proprioceptive senses to exemine whether these exercises are helpful for relieving low back pain.

\section{SUBJECTS AND METHODS}

The subjects were nonspecific lumbar pain patients aged between 19 and 65 whose duration of disease was more than three months. Fifty-four patients who understood the purpose of this study and agreed to participate were randomly allocated to a PNF exercise treatment group (26) or a stabilization exercise treatment group (28). The criteria for exclusion were: those who had structural differences in lower extremity length, a pathological finding of tumors, a history of surgery for severe trauma, a rheumatoid disease, a neurological problem, recent treatment to relieve pain or regular medication, treatment in a large hospital within the past three months, or an exercise habit of at least three days a week ${ }^{12)}$.

Prior to the exercise treatment, both the lumbar stabilization exercise group (supine, bridge, quadriped, standing position) and the PNF exercise program group (LE. flexionadduction-ext. rotation with knee flexion, sprinter, lifting) were given hyperthermia treatment for 20 minutes using a hot compress, electrotherapy using interferential treatment for 20 minutes, and deep ultrasound treatment for 10 minutes. A set of lumbar stabilization or PNF exercises comprised five repetitions. Each repetition consisted of a 10 -second exercise period and a 10 -second rest period. The subjects performed three sets of each type of exercise, four times per week for six weeks.

A visual analogue scale was used to measure the subjects' lumbar pain. The subjects marked their subjective pain level on the test paper. Ten points were given for the maximum level of pain, and zero points were given for the minimum level of pain ${ }^{13)}$.

For the measurement of trunk repositioning errors, subjects memorized the target position of flexing the trunk at 30 degrees for a few seconds, then adopted a comfortable position. Subsequently, they adopted the target position three times. The angles were measured with a digital goniometer. Swinkels \& Dolan measured the error values of the spinous process, L1, at 33.25 degrees, one third of the maximum range of the lumbar area and in another study, compared the flexion errors of the L12 ${ }^{14)}$.
Accordingly, this study measured flexion error of L12. The line connecting both the iliac crests, in other words, the fourth and fifth lumbar spinous processes, were identified, and from this point, palpation was performed in order to find the twelfth thoracic spinous process. It was marked with a pen, and its angle was measured. In order to minimize sensory stimuli from subjects' clothing during the measurement, subjects were instructed to wear simple clothing. They sat on a backless chair, on which they were able to comfortably move the legs to shoulder width, and wore a blindfold in order to block visual information.

SPSS was used for the statistical analysis of the study results. The subjective pain level and trunk position sense of the PNF exercise group and the stabilization exercise group were examined prior to treatment, three weeks after treatment, and six weeks after treatment using one-way repeated analysis of variance (ANOVA). The independent sample t-test was conducted in order to examine the differences between the two groups in terms of subjective pain level and trunk position sense. A p-value of less than 0.05 was considered to be statistically significant.

\section{RESULTS}

There were 54 subjects, with 26 in the PNF exercise group and 28 in the stabilization exercise group. There were no statistically significant differences between the two groups according to Levene's test for homogeneity of variance and a normality test $(\mathrm{p}>0.05)$ (Table 1$)$.

Table 2 shows each group's subjective pain scores according to the intervention period (Table 2). There were a statistically significant differences within the PNF exercise group and the stabilization exercise group according to treatment period $(\mathrm{p}<0.05)$, but no statistically significant differences between the two groups in terms of the VAS scores $(\mathrm{p}>0.05)$.

Table 3 displays each group's trunk repositioning errors according to the intervention period (Table 3). Both groups showed significantly different results in terms of repositioning error scores, and they also displayed statistically significant differences with each other $(\mathrm{p}<0.05)$.

\section{DISCUSSION}

The etiologies of lumbar pain include dynamic causes, such as intervertebral disk herniation of the nucleus pulposus, structural causes resulting from spinal lesion, intraperitoneal reproductive system disorders, fainting pain in the musculoskeletal system, trauma, and bad posture ${ }^{15,16)}$. Miyakoshi et al. observed that people experienced chronic lumbar pain starting in their $30 \mathrm{~s}$, and that it was associated with regenerative changes in the spine itself. They added that such changes were closely related to aging, inefficient lumbar spinal exercise, bad posture, and mental and psychological factors. They also reported that lumbar pain patients experienced functional disorders and psychological atrophy, such as depression and asthenia, because of the pain ${ }^{17}$.

There are two types of lumbar pain: acute stage lumbar pain and chronic stage lumbar pain. Lumbar pain that 
Table 1. General characteristics of subjects

\begin{tabular}{lcc}
\hline & $\begin{array}{c}\text { PNF exercise } \\
\text { Group }(\mathrm{n}=26)\end{array}$ & $\begin{array}{c}\text { Stabilizing exercise } \\
\text { group }(\mathrm{n}=28)\end{array}$ \\
\hline Age (years) & $58.0 \pm 6.3$ & $60.8 \pm 5.7$ \\
Height $(\mathrm{cm})$ & $159.6 \pm 9.0$ & $155.0 \pm 5.4$ \\
Weight $(\mathrm{kg})$ & $56.3 \pm 8.1$ & $55.8 \pm 8.1$ \\
\hline
\end{tabular}

Values are $\mathrm{M} \pm \mathrm{SE}$

Table 2. Changes in VAS pain score over the treatment period for each group

\begin{tabular}{lccc}
\hline & Pre-test & 3 weeks test & Post-test \\
\hline PNF exercise $(\mathrm{n}=26)$ & $6.50 \pm 1.14$ & $3.46 \pm 1.14$ & $1.77 \pm 0.65$ \\
Stabilizing exercise $(\mathrm{n}=28)$ & $6.18 \pm 1.12$ & $3.54 \pm 1.29$ & $1.82 \pm 0.77$ \\
\hline
\end{tabular}

(unit: score) Values are $\mathrm{M} \pm \mathrm{SE}$

Table 3. Changes in RE over the treatment period for each group

\begin{tabular}{lccc}
\hline & Pre-test & 3 weeks test & Post-test \\
\hline PNF exercise $(\mathrm{n}=26)$ & $5.12 \pm 1.33$ & $3.85 \pm 1.49$ & $2.08 \pm 1.06$ \\
Stabilizing exercise $(\mathrm{n}=28)$ & $4.07 \pm 1.25$ & $2.82 \pm 1.24$ & $2.43 \pm 1.20$ \\
\hline (unit: $^{\circ}$ ) Values are $\mathrm{M} \pm \mathrm{SE}$ & & &
\end{tabular}

stops within six weeks is acute lumbar pain and lumbar pain of a duration of twelve weeks or longer is classified as chronic lumbar pain ${ }^{18)}$. Lumbar pain reduces the activity of the cross-section muscles around the spine, and weakens muscle strength, thereby bringing about disuse atrophy. Furthermore, it decreases endurance and flexibility, which limits the range of lumbar motion.

This study examined the VAS subjective pain scores of subjects with lumbar pain, in order to examine the effects of exercise treatment on their subjective pain levels and found significant decreases in both the PNF exercise group and the stabilization exercise group. Previous studies examined the effect on pain of stabilization exercises for chronic lumbar pain and found significant differences after the exercises.

The present study compared stabilization exercises and PNF exercises, whose significance was verified. Both groups' chronic pain significantly decreased with the progression of the exercise, and without significant differences between them.

The lumbar area has conflicting aspects in functional terms, and requires postural adjustment during static and dynamic activities to adjust to the external environment. Postural adjustment is made based on the information received by the visual and somatic senses, and the vestibular system. Coordination between protagonists and antagonists needs to be made based on this information ${ }^{19)}$. Popa et al. examined the postural maintenance capabilities of lumbar pain patients and normal people and found that the patients had much lower adjustment abilities than the normal subjects $^{20)}$.

Gill et al. compared the lumbar position senses of 20 chronic lumbar pain patients and 20 normal subjects. Repositioning errors were observed with the subjects flexing at 20 degrees ten times in and upright standing position, and a four-point position on both hands and feet, and repositioning themselves. The error angles of the lumbar patients and the normal subjects were 6.71 degrees and 4.45 degrees, respectively, in the standing position, and 8.07 degrees and 5.64 degrees, respectively, in the four-point position on both hands and feet, with significantly different levels of accuracy between the two groups. The above results suggest that these two groups' proprioceptive senses differed from one another ${ }^{21)}$.

In order to examine the repositioning errors of the PNF exercise group and the stabilization exercise group, we established a target position of flexing the trunk at 30 degrees and calculated the average values of the absolute error angles. The values significantly decreased in both groups. Epecially, the reduction in the average repositioning error score was greater in the PNF exercise group than in the stabilization exercise group.

This study examined the relationship between pain and repositioning errors after performance of PNF exercises and stabilization exercises by 54 lumbar pain patients for two months beginning in August 2009. The PNF exercise group included 26 patients, and the stabilization exercise group included 28 patients. Both groups saw their trunk repositioning errors significantly decrease, but the PNF group's reduction in trunk repositioning average error was greater than that of the stabilization exercise group. In conclusion, we believe PNF exercise improves lumbar patients' proprioceptive senses and expect it to be utilized in order to relieve patients' lumbar pain and prevent its recurrence.

\section{REFERENCES}

1) Kamaz M, Kiresi D, Oguz H, et al.: CT measurement of trunk muscle 
areas in patients with chronic low back pain. Diagn Interv Radiol, 2007, 13: 144-148. [Medline]

2) Yoshihara K, Shirai Y, Nakayama Y, et al.: Histochemical changes in the multifidus muscle in patients with lumbar intervertebral disc herniation. Spine, 2001, 26: 622-626. [Medline] [CrossRef]

3) Cakir B, Richter M, Huch K, et al.: Dynamic stabilization of the lumbar spine. Orthopedics, 2006, 29: 716-722. [Medline]

4) O'Sullivan PB, Burnett A, Floyd AN, et al.: Lumbar repositioning deficit in a specific low back pain population. Spine, 2003, 28: 1074-1079. [Medline] [CrossRef]

5) Allison GT, Fukushima S: Estimating three-dimensional spinal repositioning error: the impact of range, posture, and number of trials. Spine, 2003, 28: 2510-2516. [Medline] [CrossRef]

6) Brooks VB: Motor control. How posture and movements are governed. Phys Ther, 1983, 63: 664-673. [Medline]

7) Gandevia SC, McCloskey DI, Burke D: Kinaesthetic signals and muscle contraction. Trends Neurosci, 1992, 15: 62-65. [Medline] [CrossRef]

8) Leinonen V, Maatta S, Taimela S, et al.: Impaired lumbar movemen perception in association with postural stability and motor- and somatosensory-evoked potentials in lumbar spinal stenosis. Spine, 2002, 27 975-983. [Medline] [CrossRef]

9) Radebold A, Cholewicki J, Polzhofer GK, et al.: Impaired postural control of the lumbar spine is associated with delayed muscle response times in patients with chronic idiopathic low back pain. Spine, 2001, 26: 724-730. [Medline] [CrossRef]

10) Voss DE, Knott M: Patterns of motion for proprioceptive neuromuscular facilitation. The British Journal of Physical Medicine: including its Application to Industry, 1954, 17: 191-198. [Medline]

11) Lam T, Pearson KG: The role of proprioceptive feedback in the regulation and adaptation of locomotor activity. Adv Exp Med Biol, 2002, 508:
343-355. [Medline] [CrossRef]

12) Viljanen M, Malmivaara A, Uitti J, et al.: Effectiveness of dynamic muscle training, relaxation training, or ordinary activity for chronic neck pain: randomised controlled trial. BMJ, 2003, 327: 475. [Medline] [CrossRef]

13) Scott J, Huskisson EC: Vertical or horizontal visual analogue scales. Ann Rheum Dis, 1979, 38: 560. [Medline] [CrossRef]

14) Swinkels A, Dolan P: Spinal position sense is independent of the magnitude of movement. Spine, 2000, 25: 98-104, discussion 105. [Medline] [CrossRef]

15) DePalma MJ, Ketchum JM, Saullo TR: Etiology of chronic low back pain in patients having undergone lumbar fusion. Pain Med, 2011, 12: 732-739. [Medline] [CrossRef]

16) Manchikanti L, Boswell MV, Singh V, et al.: Prevalence of facet joint pain in chronic spinal pain of cervical, thoracic, and lumbar regions. BMC Musculoskelet Disord, 2004, 5: 15. [Medline] [CrossRef]

17) Miyakoshi N, Hongo M, Kasukawa $Y$, et al.: Prevalence, spinal alignment, and mobility of lumbar spinal stenosis with or without chronic low back pain: a community-dwelling study. Pain Research and Treatment, 2011: 340629.

18) Wheeler AH: Diagnosis and management of low back pain and sciatica Am Fam Physician, 1995, 52: 1333-1341, 1347-1338.

19) Panjabi MM: The stabilizing system of the spine. Part II. Neutral zone and instability hypothesis. J Spinal Disord, 1992, 5: 390-397. [Medline] [CrossRef]

20) Popa T, Bonifazi M, Della Volpe R, et al.: Adaptive changes in postural strategy selection in chronic low back pain. Exp Brain Res, 2007, 177: 411-418.

21) Gill KP, Callaghan MJ: The measurement of lumbar proprioception in individuals with and without low back pain. Spine, 1998, 23: 371-377. [Medline] [CrossRef] 